\title{
New Opportunities for In situ Science Based on the TEAM Platform
}

\author{
D.J. Miller, ${ }^{*}$ U. Dahmen, ${ }^{* *}$ E.A. Stach *** \\ * Electron Microscopy Center, Argonne National Laboratory, Argonne, IL 60439 \\ ** National Center for Electron Microscopy, Lawrence Berkeley National Laboratory \\ *** Center for Functional Nanomaterials, Brookhaven National Laboratory
}

Among the most exciting opportunities for modern science is the capacity to study materials and processes in their natural or operating environments with the high-resolution imaging, diffraction and spectroscopy typical of electron microscopy. While transmission electron microscopes with environmental capability are already in operation and have yielded significant scientific advances, the recent development of several novel technologies offer many new opportunities for in situ TEM.

The Transmission Electron Aberration-corrected Microscope (TEAM) project pioneered several important developments, including the first realization of combined spherical $\left(\mathrm{C}_{\mathrm{s}}\right)$ and chromatic $\left(\mathrm{C}_{\mathrm{c}}\right)$ aberration correction, a new high brightness FEG, and novel fast detectors. [1] These advances provide a pathway for new opportunities for in situ science, and the first TEAM instrument is already demonstrating significant advance for atomic-scale imaging during in situ experimentation. With $\mathrm{C}_{\mathrm{s}} / \mathrm{C}_{\mathrm{c}}$ aberration correction, TEAM offers sub-Ångstrom resolution in TEM mode even at low $(80 \mathrm{keV})$ accelerating voltage that enables real-time observation of the motion and rearrangement of individual atoms at isolated edges in a single atomic layer of graphene (Fig. 1). [2] Studies of such atomic motion have not been possible thus far using scanning probe microscopies such as STM.

Beyond resolution, one of the most promising opportunities offered by $\mathrm{C}_{\mathrm{s}} / \mathrm{C}_{\mathrm{c}}$ correction is the possibility to open up the pole piece gap to increase the experimental space around a sample without significantly compromising resolution [3], as illustrated in Fig. 2. This possibility has inspired several new concepts for improving our capabilities for in situ science. For example, this experimental space can be used to incorporate enhanced analytical capabilities, additional probes, and enhanced environment around a sample. Our concept for an in situ version of TEAM, $i$ TEAM, would integrate $\mathrm{C}_{\mathrm{s}}$ probe correction, post specimen $\mathrm{C}_{\mathrm{s}} / \mathrm{C}_{\mathrm{c}}$ aberration correction, and high efficiency and novel spectroscopies within a large-gap $(\approx 1 \mathrm{~cm})$ environmental TEM. An example of one implementation, the incorporation of a high collection efficiency X-ray detector [4] incorporated into a wide-gap instrument, is shown in Fig. 3. This configuration would allow researchers to coordinate morphological observations and high spatial resolution spectroscopy during in situ experiments. Importantly, $\mathrm{C}_{\mathrm{s}} / \mathrm{C}_{\mathrm{c}}$ correction brings the additional benefit of improving the resolution for imaging of "thick" samples where imaging is degraded due to chromatic losses. Such effects will be routinely encountered for in situ environmental studies where the in situ environment itself contributes to chromatic losses and for which it is important to design experiments that reliably reproduce "bulk" situations [5], placing a constraint on the degree to which one can work on "thin" samples.

A wide-gap instrument also offers the opportunity to integrate other experimental probes to provide information that can complement the insight gained from electron microscopy. For example, the integration of optical spectroscopy into the electron microscope can further expand our in situ capabilities by providing high energy-resolution vibrational spectroscopy. Although in principle such measurements can be done using EELS, they are rarely conducted in a TEM due to the extremely high energy-resolution $(\sim 10 \mathrm{meV})$ required. In addition, a wide-gap $\mathrm{C}_{\mathrm{s}} / \mathrm{C}_{\mathrm{c}}$ corrected instrument offers advantages for high-resolution cryo microscopy of interfaces between soft and 
hard materials, a concept termed "SofTEAM." A more ambitious concept involves the integration of both electron microscopy and synchrotron radiation beam lines. This "XTEAM" concept would rely on $\mathrm{C}_{\mathrm{s}} / \mathrm{C}_{\mathrm{c}}$ correction to provide high resolution for a wide gap lens designed to accommodate both types of probes. The result would be an instrument that combines the atomistic characterization of $\mathrm{STEM} / \mathrm{TEM}$ with ensemble-averaged spectroscopy provided by $\mathrm{x}$-rays.

Building on the success of TEAM, together these capabilities offer the possibility to revolutionize our ability to study materials in "real world" environments with the resolution and rich information that makes electron microscopy so powerful.

\section{References}

[1] U. Dahmen et al., Phil. Trans. Roy. Soc. A 367 (2009) 3795

[2] C.O. Girit, et. al., Science 323 (2009) 1705.

[3] B. Kabius, et. al., Journal of Electron Microscopy 58(3) (2009) 147.

[4] N.J. Zaluzec, Microscopy Today 17 (4) (2009) 56, and US Patents: \#6548810, \# 61317847.

[5] M.J. Williamson, et. al., Nature Materials 2 (2003) 532.

[6] TEAM and this research were supported by the U.S. Department of Energy Office of Science, Basic Energy Sciences under contracts DE-AC02-06CH11357 (ANL EMC), DE-AC02 05CH11231 (LBL NCEM), and DE-AC02-98CH10886 (BNL CFN).
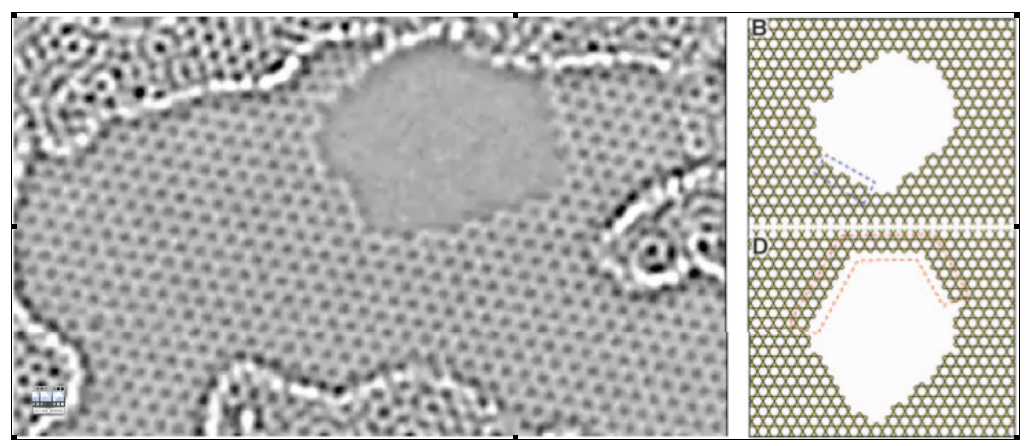

FIG. 1. Atomic-resolution image (a) and edge configurations $(b, c)$ showing the rearrangement of individual atoms at the edge of a hole in a single layer of graphene under electron irradiation. [2]

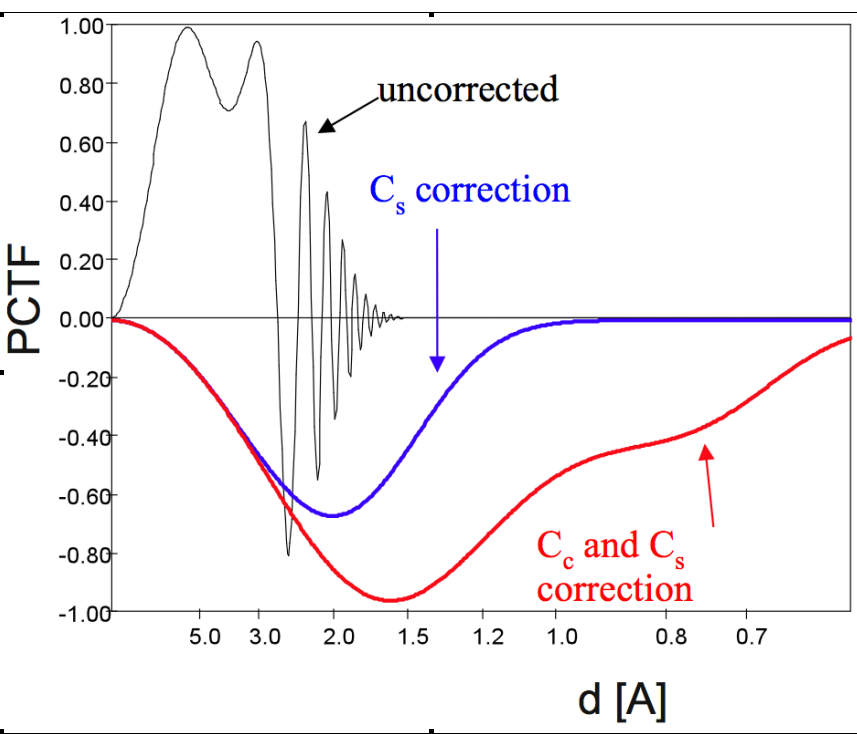

FIG. 2. Calculated contrast transfer/damping envelopes for a wide-gap $(10 \mathrm{~mm})$ objective lens in the uncorrected state, with $\mathrm{C}_{\mathrm{s}}$ correction, and with $\mathrm{C}_{\mathrm{s}} / \mathrm{C}_{\mathrm{c}}$ correction. [see ref. 3]

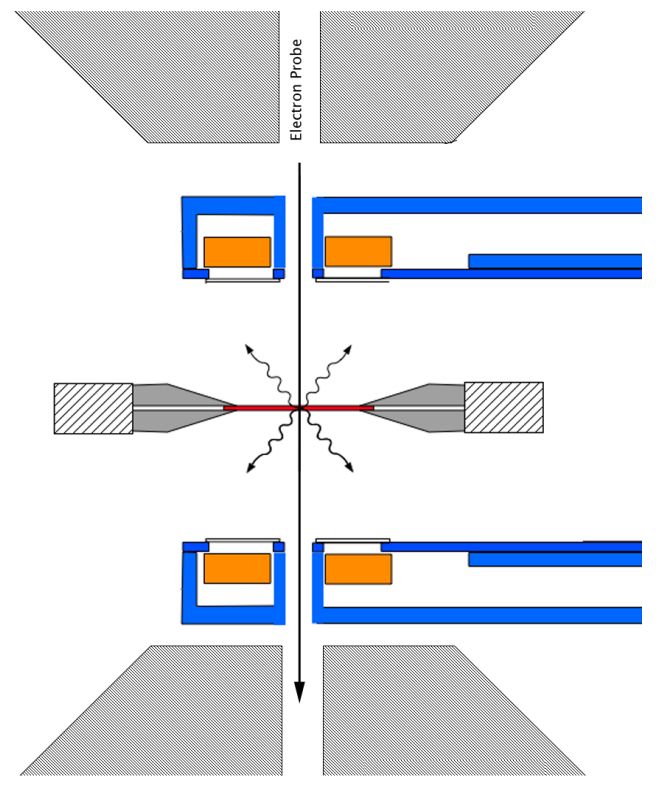

FIG. 3. Illustration (not to scale) of a high collection efficiency double X-ray detector incorporated into a wide-gap instrument. [4] 